\title{
Impaired Gamma-Band Activity during Perceptual Organization in Adults with Autism Spectrum Disorders: Evidence for Dysfunctional Network Activity in Frontal-Posterior Cortices
}

\author{
Limin Sun, ${ }^{1}$ Christine Grützner, ${ }^{1}$ Sven Bölte, ${ }^{2,3}$ Michael Wibral, ${ }^{4}$ Tahmine Tozman, ${ }^{5}$ Sabine Schlitt, ${ }^{2}$ Fritz Poustka, ${ }^{2}$ \\ Wolf Singer, ${ }^{1,6,7}$ Christine M. Freitag, ${ }^{2}$ and Peter J. Uhlhaas ${ }^{1,7}$ \\ ${ }^{1}$ Department of Neurophysiology, Max-Planck Institute for Brain Research, 60528 Frankfurt am Main, Germany, ${ }^{2}$ Department of Child and Adolescent \\ Psychiatry, Psychosomatics and Psychotherapy, Goethe University Frankfurt am Main, 60528 Frankfurt am Main, Germany, ${ }^{3}$ Department of Women's and \\ Children's Health, Astrid Lindgren Children's Hospital, Center of Neurodevelopmental Disorders (KIND), Karolinska Institutet, 17176 Stockholm, Sweden, \\ ${ }^{4}$ MEG Unit, Brain Imaging Center, Johann Wolfgang Goethe University, 60528 Frankfurt am Main, Germany, ${ }^{5}$ Department of Educational Psychology, \\ Goethe University Frankfurt am Main, 60325 Frankfurt am Main, Germany, ${ }^{6}$ Frankfurt Institute for Advanced Studies, Johann Wolfgang Goethe University, \\ 60438 Frankfurt am Main, Germany, and ${ }^{7}$ Ernst-Strüngmann Institute for Neuroscience, in Cooperation with Max-Planck Society, Frankfurt am Main,
} 60528, Germany

Current theories of the pathophysiology of autism spectrum disorders (ASD) have focused on abnormal temporal coordination of neural activity in cortical circuits as a core impairment of the disorder. In the current study, we examined the possibility that gamma-band activity may be crucially involved in aberrant brain functioning in ASD. Magneto-encephalographic (MEG) data were recorded from 13 adult human participants with ASD and 16 controls during the presentation of Mooney faces. MEG data were analyzed in the 25-150 $\mathrm{Hz}$ frequency range and a beamforming approach was used to identify the sources of spectral power. Participants with ASD showed elevated reaction times and reduced detection rates during the perception of upright Mooney faces, while responses to inverted stimuli were in the normal range. Impaired perceptual organization in the ASD group was accompanied by a reduction in both the amplitude and phase locking of gamma-band activity. A beamforming approach identified distinct networks during perceptual organization in controls and participants with ASD. In controls, perceptual organization of Mooney faces involved increased $60-120 \mathrm{~Hz}$ activity in a frontoparietal network, while in the ASD group stronger activation was found in visual regions. These findings highlight the contribution of impaired gamma-band activity toward complex visual processing in ASD, suggesting atypical modulation of high-frequency power in frontoposterior networks.

\section{Introduction}

Autism spectrum disorders (ASD) are predominantly genetically mediated syndromes (Freitag et al., 2010; Geschwind, 2011) characterized by impairments in reciprocal social interaction and communication alongside with restricted and repetitive behaviors (American Psychiatric Association, 2000). In addition to these clinical symptoms, ASD involve a wide range of cognitive alterations including changes in basic sensory processing and

\footnotetext{
Received March 5, 2012; revised May 21, 2012; accepted May 23, 2012.

Author contributions: C.G. and P.J.U. designed research; C.G., S.B., T.T., S.S., F.P., and C.M.F. performed research;

L.S., C.G., M.W., and P.J.U. analyzed data; W.S. and P.J.U. wrote the paper.

This work was supported by the Max-Planck Society and the LOEWE NeFF-Grant Neuronale Koordination Forschungsschwerpunkt Frankfurt.

Correspondence should be addressed to Dr. Peter J. Uhlhaas, Department of Neurophysiology, Max-Planck Institute for Brain Research, Deutschordenstrasse 46, Frankfurt am Main, 60528, Germany. E-Mail: peter. uhlhaas@brain.mpg.de.

DOI:10.1523/JNEUROSCI.1073-12.2012

Copyright $\odot 2012$ the authors $\quad 0270-6474 / 12 / 329563-11 \$ 15.00 / 0$
}

higher level cognitive processes (Baron-Cohen et al., 1985; Happé, 1996; Hill, 2004).

Evidence is emerging that cognitive alterations as well as clinical symptoms may arise from impaired coordination of distributed neuronal activity (Uhlhaas and Singer, 2007; Minshew and Keller, 2010; Schipul et al., 2011). In support of this hypothesis, several studies have demonstrated altered functional connectivity of blood oxygenation level-dependent responses during a range of cognitive tasks in ASD (Castelli et al., 2002; Just et al., 2004; Kleinhans et al., 2008).

Although informative, functional magnetic resonance imaging (fMRI) confers only limited insights into the timing of neuronal responses because of the slow temporal resolution relative to electroencephalography or magneto-encephalography (EEG/ MEG). EEG and MEG allow the measurement of electrophysiological signals with millisecond precision, a timescale that is fundamental for precise neural coding (Havenith et al., 2011). Specifically, oscillatory activity at low- and high-frequency ranges has been related to a range of cognitive and perceptual processes 
(Rodriguez et al., 1999; Uhlhaas et al., 2009). Of special importance are oscillations in the gamma-band range $(30-200 \mathrm{~Hz}$ ) range as they are particularly suited for establishing precise synchronization in local circuits (Fries, 2009).

Preliminary evidence suggests that ASD may be associated with changes in gamma-band oscillations. In the auditory domain, reduced entrainment to auditory stimulation at $40 \mathrm{~Hz}$ in participants with ASD (Wilson et al., 2007) as well as their firstdegree relatives (Rojas et al., 2011) has been demonstrated. In contrast, during visual perception there is evidence for both hyperactivity and hypoactivity of gamma-band oscillations (Grice et al., 2001; Brown et al., 2005; Milne et al., 2009; Isler et al., 2010; Stroganova et al., 2012), raising the question of the link between high-frequency oscillations and perceptual dysfunctions in the disorder.

To clarify the role of gamma-band activity in perceptual dysfunctions and in the pathophysiology of ASD, we investigated spectral power in the 25-150 Hz frequency range during perceptual organization of Mooney faces in an adult sample of high-functioning participants with ASD $(n=13)$ and matched healthy control participants $(n=16)$. We used MEG because the magnetic field can be measured undisturbed by tissue inhomogeneities, which results in an improved detectability of high-frequency oscillations and enhanced localization accuracy compared with EEG (Kaiser and Lutzenberger, 2005). Specifically, we focused on the high $(60-120 \mathrm{~Hz})$ gamma-band range because previous work had demonstrated that the $60-120 \mathrm{~Hz}$ band is critical for perceptual organization of Mooney faces (Grützner et al., 2010).

Based on current pathophysiological models of ASD that have discussed a dysfunction in the excitation-inhibition balance (E/I balance) (Rubenstein and Merzenich, 2003) as well as atypical modulation in frontoposterior networks (Minshew and Keller, 2010; Schipul et al., 2011), we expected reduced gamma-band power during perceptual processing in participants with ASD as well as abnormal modulation of high-frequency activity in frontal, parietal, and sensory cortices.

\section{Materials and Methods \\ Participants}

Thirteen participants with ASD (11 males, mean age: 30.3 years) were recruited from the Department of Child and Adolescent Psychiatry, Psychosomatics, and Psychotherapy of the Goethe University at Frankfurt/M. Inclusion criteria were a clinical diagnosis of autism, Aspergers disorder, or pervasive developmental disorder not otherwise specified (PDD-NOS) according to DSM-IV (American Psychiatric Association, 2000 ), age $>18$ years and IQ $>70$. The clinical diagnosis was corroborated using the German form of the Autism Diagnostic InterviewRevised (Schmötzer et al., 1993; Lord et al., 1994) and the Autism Diagnostic Observation Schedule (Lord et al., 2000). General IQ was assessed with the standard progressive matrices (Raven, 1948).

In addition, 16 healthy controls (12 males, mean age: 29.7$)$ were recruited from the local community and screened for psychopathology with the German version of Structured Clinical Interview for DSM-IV-R Non-Patient Edition (Sass et al., 2003). There were no significant differences between participants with ASD and controls in IQ, sex distribution, and age (Table 1). Written informed consent was obtained from all par-
Table 1. Mean, SDs, and mean differences for genders, ages, and IQ scores of controls and participants with ASD

\begin{tabular}{|c|c|c|c|c|c|c|}
\hline & \multicolumn{2}{|c|}{$\begin{array}{l}\text { Healthy controls } \\
(n=16)\end{array}$} & \multicolumn{2}{|c|}{$\begin{array}{l}\text { ASD group } \\
(n=13)\end{array}$} & \multicolumn{2}{|l|}{ Mean differences } \\
\hline & Mean & SD & Mean & SD & $\chi^{2} / t$ value & $p$ value \\
\hline Gender (M/F) & $12 / 4$ & & $11 / 2$ & & $\chi^{2}(1)=0.55$ & 0.446 \\
\hline Age (years) & 29.69 & 6.88 & 30.31 & 9.60 & $t(27)=0.20$ & 0.847 \\
\hline SPM IQ & 116.06 & 8.42 & 107.75 & 14.63 & $t(27)=-1.76$ & 0.097 \\
\hline
\end{tabular}

ASD, autism spectrum disorders; SPM, standard progressive matrices. 
Table 2. Means, SDs, and mean differences for behavioral performance in controls and participants with ASD

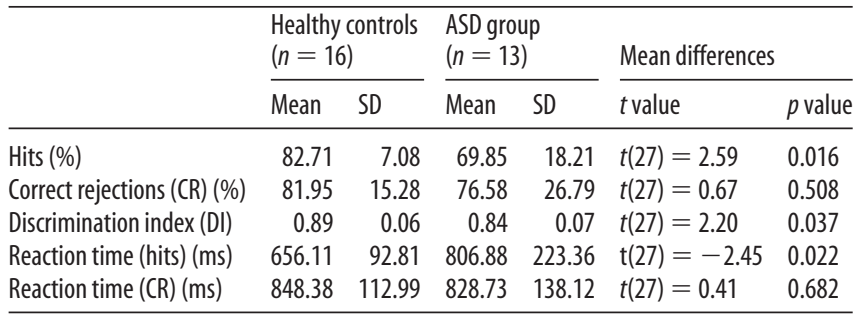

ASD, autism spectrum disorders.

\section{Analysis of sensor-level spectral power changes}

Time-frequency representations (TFRs) were computed by means of Morlet wavelets with a width of five cycles per wavelet at center frequencies between 25 and $150 \mathrm{~Hz}$, in $1 \mathrm{~Hz}$ steps. For a statistical analysis of the event-related changes in gamma-band power, we focused on the face condition and performed three tests. First, we tested changes in gammaband power within each group and compared the raw power during stimulus presentation (50-350 ms poststimulus) to the raw power during baseline ( -350 to $-50 \mathrm{~ms}$ prestimulus) using permutation tests based on the dependent-samples $t$-metrics. Next, we compared gammaband power between controls and patients for the interval between 50 and $350 \mathrm{~ms}$ poststimulus using permutation tests based on the independent-samples $t$-metric. The betweengroup analysis was computed on absolute power difference values; that is, the raw baseline power was subtracted from the raw task power for each subject. All statistical tests were computed as mass-univariate tests for all frequency bins in the frequency range of 25-140 $\mathrm{Hz}$ and included all channels. To correct for the arising multiple-comparison problem we used cluster-based statistical analysis (Maris and Oostenveld, 2007) with a criterion of $p<0.05$.

In addition to estimating power values, we also computed intertrial phase coherence (ITPC) (Delorme and Makeig, 2004) across all sensor groups for low-frequency delta (1-3 $\mathrm{Hz})$, theta $(4-7 \mathrm{~Hz})$, and alpha $(8-12 \mathrm{~Hz})$ bands as well as for high-frequency activity (beta band [13-25 Hz] and low [25-60 Hz] and high $[60-140 \mathrm{~Hz}]$ gamma bands). This analysis approach was used to distinguish transient oscillatory activity associated with the on

tation was controlled using the Presentation software package (Neurobehavioral Systems).

\section{MR data acquisition}

Structural MR images were obtained with a $3 \mathrm{~T}$ Siemens Allegra scanner, using a CP head coil for radio frequency transmission and signal reception. A 3D MPRAGE sequence ( 160 slices, voxel size $=1 \times 1 \times 1 \mathrm{~mm}$, $\mathrm{FOV}=256, \mathrm{TR}=2300, \mathrm{TE}=3.93)$ was used for the structural scan. Before the structural scan, vitamin E pills were placed at the nasion and 1 $\mathrm{cm}$ anterior to the tragus of the left and right ear to allow for coregistration of the MEG and MRI data.

\section{$M E G$ recording}

Acquisition. MEG signals were recorded with a 275-channel whole-head system (Omega 2005; VSM MedTech with $600 \mathrm{~Hz}$ sample rate and processed with a digital bandpass filter (fourth order Butterworth filter, low-frequency cutoff $0.5 \mathrm{~Hz}$ and high-frequency cutoff $150 \mathrm{~Hz}$ ). Before each run, the head position was relocalized according to three coils placed on the nasion and $1 \mathrm{~cm}$ anterior to the tragus of the left and right ear. The head movement was kept $<5 \mathrm{~mm}$. Runs with larger movements were discarded. Behavioral responses were recorded using a fiberoptic response pad (lumitouch; Photon Control) of the presentation computer and fed back to MEG acquisition system as an additional channel.

\section{MEG data processing}

MEG data were analyzed using the FieldTrip open source MATLAB Toolbox (http://www.ru.nl/fcdonders/fieldtrip/). Trials were defined from the continuously recorded MEG from - 1000 to $1000 \mathrm{~ms}$ with respect to the onset of the visual stimulus and classified according to the two experimental conditions: the face condition, containing trials with upright stimuli, and the no-face condition, containing trials with inverted-scrambled stimuli. Only data with correct responses were considered for all further analyses.

Data epochs contaminated by eyeblinks, muscle activity, or jump artifacts in the SQUIDs were discarded using automatic artifact detection and rejection routines provided by the FieldTrip software. Nonartifact trials were baseline corrected by subtracting the mean amplitude during an epoch ranging from -500 to $-100 \mathrm{~ms}$ before stimulus onset. and off responses from induced, nonphase-locked oscillations.

Reconstruction of sources of oscillatory sensor-level components

To localize the sources underlying spectral power changes, we used a linearly constrained minimum variance beamformer (Van Veen et al., 1997) as implemented in FieldTrip. This technique estimates the source power for each voxel in the brain by constructing an adaptive spatial filter that connects each voxel with the MEG sensors. The spatial filter at each cortical location is constructed such that the sensitivity to a source at the target location is fixed at unit gain, while the total output power of the filter is minimized, thus minimizing cross talk between sources. For the computation of the forward model, we used a realistic single-shell volume conductor model (Nolte and Dassios, 2005). To obtain the shape parameters for this model, a segmentation of each participant's individual MRI was performed using SPM2 (http://www.fil.ion.ucl.ac.uk/spm).

We first filtered the data in the low $(25-60 \mathrm{~Hz})$ and high $(60-120 \mathrm{~Hz})$ gamma-band frequency ranges, respectively, and computed the covariance matrix of the filtered data. We used a narrower frequency range for the high gamma band in this analysis $(60-120 \mathrm{~Hz})$ compared with the sensor-level analyses (up to $140 \mathrm{~Hz}$ ) to minimize the difference in bandwidth for the beamformer analyses in the low and high gamma frequency ranges. Thus, for the source reconstruction of high gamma-band power, we focused on the frequency window that had shown the most pronounced task-related effects in the high gamma-band range at sensor level.

For each of the two frequency ranges, the covariance matrix was then computed for all face trials, for both patients and controls, separately for baseline $(-350$ to $-50 \mathrm{~ms})$ and activation epochs $(50-350 \mathrm{~ms})$. The covariance matrices for baseline and activation epochs were averaged to allow for the computation of common filters. We used a common filter throughout the analyses because computing separate filters for task and baseline may have introduced differences in the beamformer filters due to changes in the number of active sources. Common filters then enabled a statistical test of the hypothesis that source power is changed by a stimulus. In contrast, when computing separate sets of filters for task and baseline, the hypothesis to be tested is that either source power changes or 


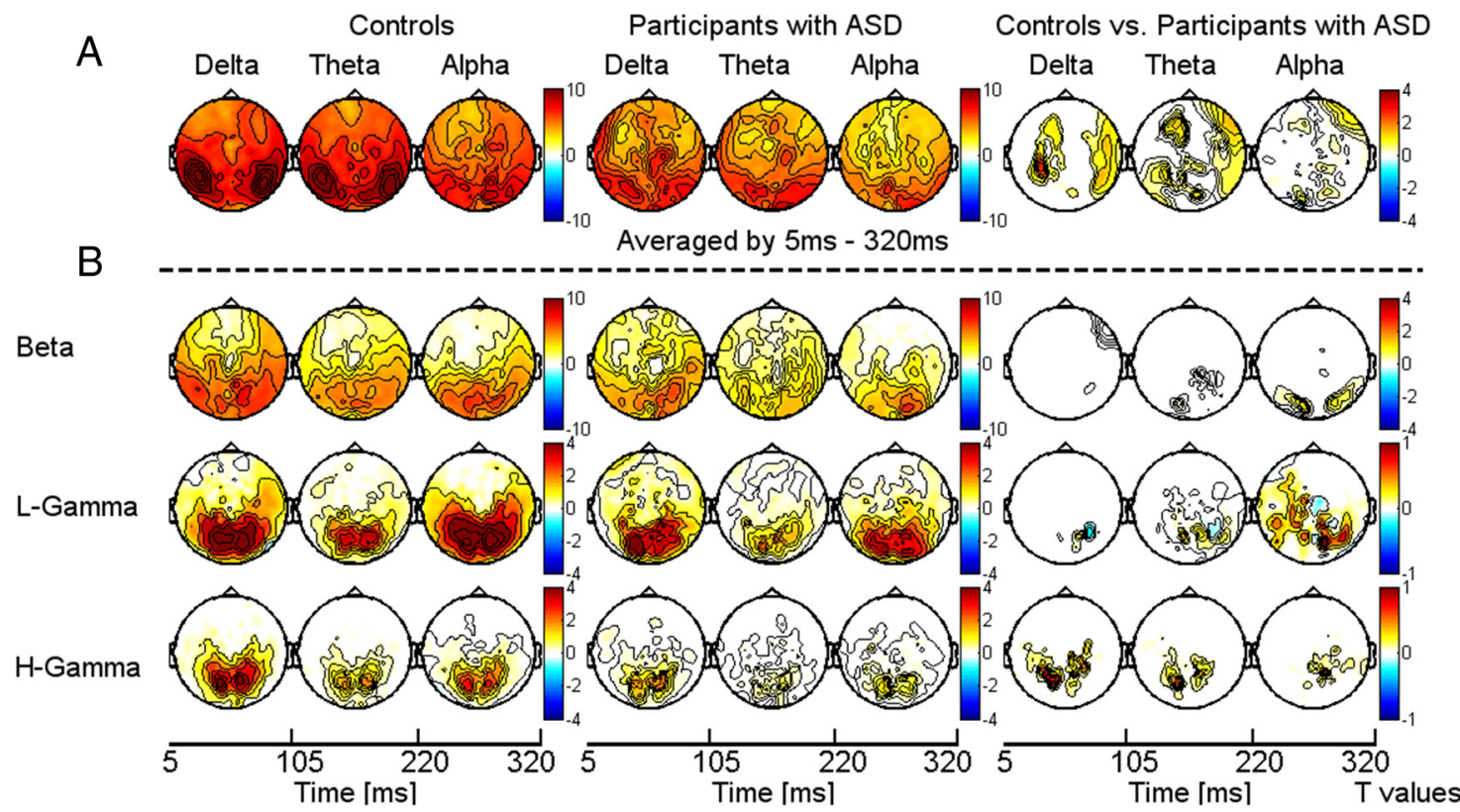

Figure 3. Topography and statistical analysis of ITPC for low-frequency activity (delta, theta, and alpha bands) $(\boldsymbol{A}$ ) averaged between 5 and $320 \mathrm{~ms}$ and high frequencies (beta band and low and high gamma band) (B) for controls (left) and probands with ASD (middle). Right panel displays the statistical differences between the ASD group and controls. ITPC values are expressed as relative change to the baseline period and corrected for multiple comparisons through a cluster-based test statistic.

beamformer filters differ between task and baseline because of a potentially changing number of active sources between task and baseline (Nieuwenhuis et al., 2008). Source power was subsequently estimated for each grid location by projecting the sensor data through the common filters for each trial separately for task and baseline.

For a statistical analysis of the source power, we first normalized the single participant and condition source power estimate to $\mathrm{MNI}$ space. At the source power level, we then performed a nonparametric ANOVA using a $2 \times 2$ design with the factors phase (task vs baseline) and group (participants with ASD vs controls). Evaluated contrasts were task versus baseline and the phase versus group by task versus baseline interaction effect. All of the statistical tests were performed using Monte Carlo permutation tests and multiple comparisons were corrected with a cluster-based test statistic (Maris and Oostenveld, 2007). Alternatively, parametric testing could have been used but the source power residuals could not be assumed to be normally distributed because of the intergroup comparisons performed. Permutation tests provide higher accuracy in this case.

In the final step, we identified those brain regions that were specifically involved in dysfunctional perceptual organization of Mooney faces through contrasting high gamma-band activity to face versus no-face stimuli between the ASD group versus controls (interaction effect). To this end, we entered the baseline-corrected data into a nonparametric ANOVA (Anderson and ter Braak, 2003) involving a $2 \times 2$ design with factors percept (face vs no-face condition) and group (ASD group vs controls). We focused on the interaction effect as this was the only term relevant for the question of which network of gamma-band sources contributed uniquely to perceptual organization deficits in participants with ASDs.

Finally, in the description of source results and their interpretation, we did not include activations in deep brain structures, such as the thalamus, because it is currently unclear whether MEG-based source reconstruction techniques are suited to study deep brain structures (Papadelis et al., 2009). Therefore, such sources have been highlighted in Figure 9 and will not be discussed.

Correlations between behavioral responses and source gamma-band power

To investigate the relationship between gamma-band power and behavior, we computed correlations between source power in the $60-120 \mathrm{~Hz}$ frequency range and reaction times, detection rates, and the discrimination index $\left(\mathrm{A}^{\prime}\right)$ for the ASD group and controls separately. Correlations were obtained in the following two steps. Dependent $t$ tests (task source power vs baseline source power) were applied to each participant with the cluster-based nonparametric permutation test described above and 
A Controls

Participants with ASD

Controls vs. Participants with ASD

\section{L-Gamma H-Gamma}

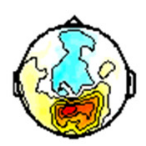

L-Gamma H-Gamma

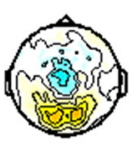

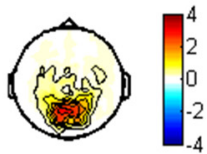

L-Gamma H-Gamma

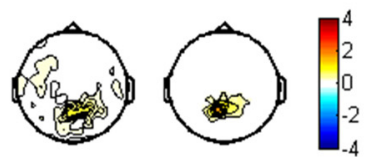

Averaged by $5 \mathrm{~ms}-320 \mathrm{~ms}$
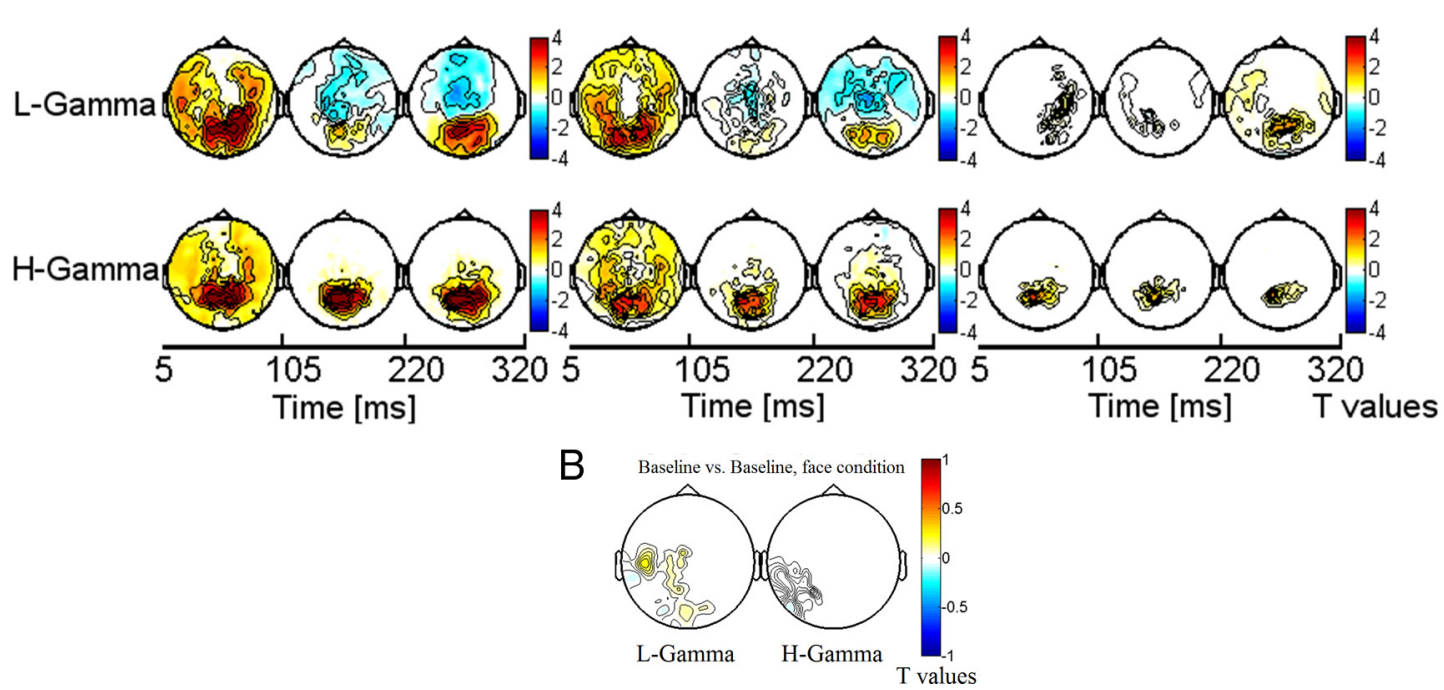

Figure 5. A, Statistical analysis of power changes in response to upright Mooney faces for controls (left), participants with ASD (middle), and for the difference between controls and participants with ASD (right). The topographies display significant differences between the face condition and the $0.5 \mathrm{~s}$ prestimulus baseline, separately for the lower $(25-60 \mathrm{~Hz})$ and the higher $(60-120 \mathrm{~Hz})$ gamma band. The effect is masked by the significance map derived from a cluster-based test statistic. Left and middle, Red denotes higher activation during stimulus presentation compared to baseline, whereas blue denotes less activation during stimulus presentation compared to baseline. Right, In the topographies (difference for the face condition between controls and participants with ASD) red denotes stronger activation for controls compared to participants with ASD, whereas blue represents stronger activation in participants with ASD relative to controls. Topographies in the top row show the averaged spectral power for the $5-320 \mathrm{~ms}$ period, whereas the topographies in the middle row show spectral values for the three time windows (on response, induced window, and offset response) separately. $\boldsymbol{B}$, Differences in baseline activity between controls and participants with ASD.

applied to the whole brain. If a $t$ value for one grid point was significant across all participants, this grid point (unit) was kept for further analysis. In the next step, each unit was then used to compute correlations with reaction times, detection rates, and the discrimination index $\mathrm{A}^{\prime}$. Only significant correlations were selected that met an $\alpha$-level of 0.05 with Bonferroni correction (Curtin and Schulz, 1998). Finally, the low spatial resolution of the correlation map was interpolated with a spline function in SPM8 to be compatible with the standard brain template for display purposes.

\section{Results}

\section{Behavioral results}

We analyzed the percentage of correct responses as well as reaction times for the face and the no-face condition (Table 2). Furthermore, we computed the discrimination index $A^{\prime}$ (Grier, 1971). The ASD group detected significantly fewer faces than controls $(t=2.589, p=0.05)$ and had longer reaction times $(t=$ $-2.445, p=0.05)$. However, responses to stimuli in the no-face condition in participants in the ASD group were in the normal range (correct responses: $t=0.672, p=0.50$; reaction times: $t=$ $0.414, p=0.68$ ). The significant difference between groups in $\mathrm{A}^{\prime}$ $(t=2.201, p=0.05)$ confirmed that controls had a better discrimination performance.

\section{ITPC analysis}

The analysis of ITPC values revealed prominent increases in the low gamma-band range during an early (5-120 ms) and a later time window (220-320 ms; Fig. 2), which likely reflected tran- sient activity related to the on and offset response of the stimulus. To differentiate spectral power related to transient activity from induced power, we used the gradient of ITPC values to define evoked activity based on the averaged power over all channels and participants in the low gamma band. Accordingly, we defined three time windows: (1) an early evoked time window (onset response: 5-105 ms), (2) an induced period (105-220 ms), and (3) a second evoked window (offset response: $220-320 \mathrm{~ms}$ ).

In both controls and participants with ASD, gamma-band ITPC was focused over occipitoparietal sensors (Fig. 3B). Compared with controls, ASD probands were characterized by significantly reduced ITPC values in the low and high gamma bands over parietal and central sensors (Fig. 2). In addition to ITPC deficits in the gamma-band range, we also found significant lower ITPC values in the lower delta, theta, and alpha bands in the ASD group (Fig. 3A).

\section{Gamma-band power at sensor level}

After artifact correction, the mean number of trials in the face condition in controls was 138 and 108 trials in the ASD group. In the no-face condition, there were 69 trials for participants with ADS and 71 for control participants. To correct for the higher number of trials in the face condition in controls relative to participants with ASD, we adjusted the number of trials in this group by randomly selecting 30 trials from each participant.

In controls, gamma-band activity was characterized by a taskrelated increase between 50 and $350 \mathrm{~ms}$ after stimulus onset with 
A Controls: task vs. baseline (face condition), $25-60 \mathrm{~Hz}$

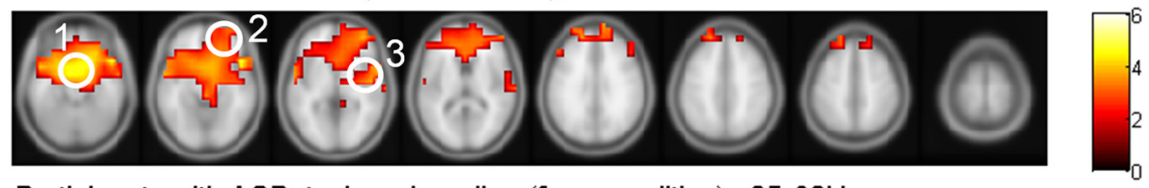

B Participants with ASD: task vs. baseline (face condition), $25-60 \mathrm{~Hz}$

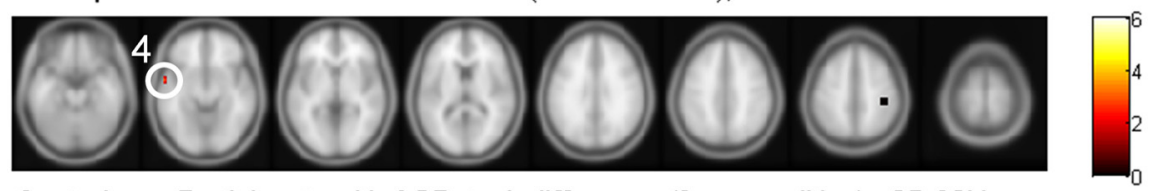

C Controls vs. Participants with ASD: task difference (face condition), $25-60 \mathrm{~Hz}$

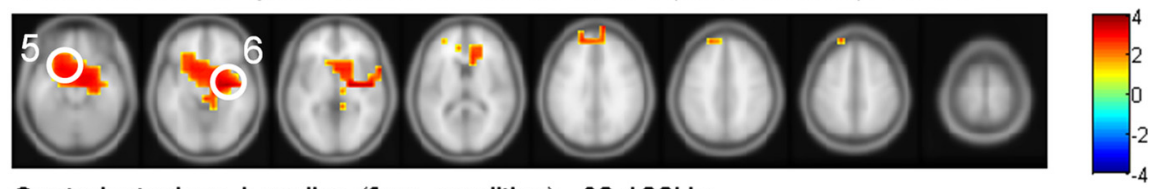

D Controls: task vs. baseline (face condition), $60-120 \mathrm{~Hz}$

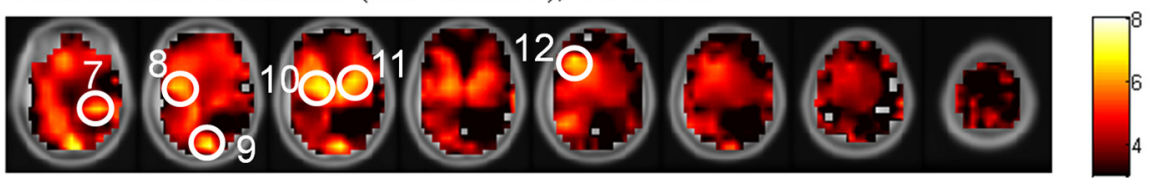

E Participants with ASD: task vs. baseline (face condition), $60-120 \mathrm{~Hz}$

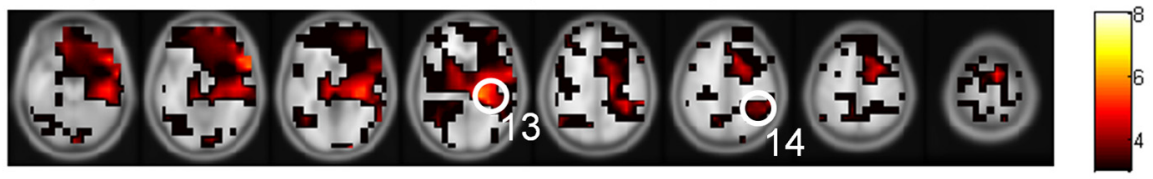

F Controls vs. Participants with ASD: task difference (face condition), $60-120 \mathrm{~Hz}$
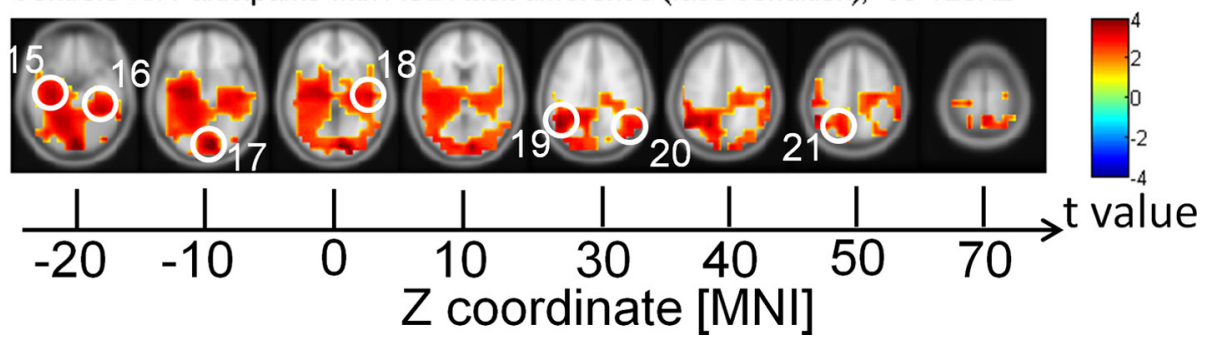

Figure 6. Source power in the face condition for controls and participants with ASD. $A, B, D, E$, Red clusters represent stronger activation in the face condition compared to baseline for controls and participants with ASD, whereas blue clusters represent stronger activation in the baseline relative to the task. $C, F$, Correspond to the differences between controls and participants with ASD in the face condition, where red clusters denote stronger activation in controls compared to participants with ASD and blue clusters denote stronger activation in participants with ASD compared to controls. Note that $p$ values are calculated by cluster-based statistical analysis. 1, MeFG; 2, MFG; 3, STG; 4, MTG; 5, IFG; 6, insula; 7, FusG; 8, STG; 9 , lingual gyrus; 10 and 11, lentiform nucleus*; 12 , MFG; 13, insula; 14, IPL; 15, parahippocampal gyrus; 16, FusG; 17, lingual gyrus; 18, STG; 19 and 20, supramarginal gyrus; 21, precuneus. *Excluded from analysis.

two prominent gamma-band peaks $\sim 50$ and $250 \mathrm{~ms}$ in the 25$140 \mathrm{~Hz}$ frequency range (Fig. 4A). We observed sustained gamma-band activity between 100 and $300 \mathrm{~ms}$, mainly between 60 and $120 \mathrm{~Hz}$. The statistical analysis of poststimulus activity in controls (task vs baseline) revealed a significant increase in lowand high-frequency gamma-band power over parieto-occipital channels. This power increase was accompanied by a significant reduction over frontocentral sensors in the low gamma-band range, which was only evident in the control group (Fig. 4A).

Participants with ASD were characterized by significantly reduced gamma-band activity over parieto-occipital sensors (Fig. $5 A$ ). The effect size for the deficit in high gamma-band activity over parieto-occipital sensors in the ASD group was large $(d=$ 1.25). Similarly, the deficit in the $25-60 \mathrm{~Hz}$ frequency reached a value of $d=0.97$.

The analysis of transient and induced time windows showed that the reduction in high gamma-band oscillations involves both the evoked activity related to the on and off response as well as induced power, while for the $25-60 \mathrm{~Hz}$ activity the deficit was only associated with the offset response (Fig. 5B).

\section{Baseline analysis}

To exclude that effects were solely driven through differences in baseline activity, we also examined baseline activity before stimulus onset in controls and participants with ASDs, which could potentially bias differences task-related activity. Controls showed a small increase in baseline power values in the $25-60 \mathrm{~Hz}$ range relative to participants with ASD (Fig. $5 B$ ). In the $60-120 \mathrm{~Hz}$ frequency range, there was no difference in baseline activity between groups.

Spatiotemporal reconstruction of gamma-band activity during the face condition

In controls, the strongest activation in the high gamma band (task vs baseline) was observed in frontal, temporal, and visual regions. Specifically, higher gamma-band activity was found in 
the lingual gyrus, the fusiform gyrus (FusG), the superior temporal gyrus (STG), and the left middle frontal gyrus (MFG) (Fig. 6). In the ASD group, source activity was generally reduced and confined to the right inferior parietal lobe (IPL) and the insula (Fig. 6). See Table 3 for anatomical locations and MNI coordinates.

In the lower gamma band, face stimuli in the control group elicited extended source activity in frontal areas, with a peak in the right frontal gyrus, MFG, medial frontal gyrus (MeFG), and STG. In contrast, in participants with ASD, lower gamma-band power sources involved only a small cluster in the left middle temporal gyrus (MTG).

Differences in source power between controls and participants with ASD revealed widespread deficits in the ASD group, which were particularly prominent in the $60-120 \mathrm{~Hz}$ frequency range (Fig. 6). In the high gamma band, reduced source power in the ASD group was localized to the FusG, the lingual gyrus, and STG of the right hemisphere. Clusters of reduced activity in the

Table 3. Anatomic locations and MNI coordinates for the sources of high and low gamma-band activity in controls and participants with ASD

\begin{tabular}{lllrrr} 
& & & MEG (MNI) & \multicolumn{2}{c}{ Coordinates } \\
\cline { 5 - 6 } Cluster & Anatomic location & $t$ value & $x$ & \multicolumn{1}{c}{$y$} & \multicolumn{1}{c}{$z$} \\
\hline 1 & Right MeFG & 4.53 & 6 & 12 & -20 \\
2 & Right MFG & 3.17 & 26 & 48 & -10 \\
3 & Right STG & 3.37 & 62 & 6 & 0 \\
4 & Left MTG & 3.27 & -58 & 0 & -10 \\
5 & Left IFG & 2.67 & -14 & 20 & -20 \\
6 & Right insula & 3.31 & 38 & -10 & -6 \\
7 & Right FusG & 6.50 & 36 & -40 & -20 \\
8 & Left STG & 4.96 & -48 & -12 & -8 \\
9 & Right lingual gyrus & 7.42 & 10 & -90 & -10 \\
10 & Left lentiform nucleus & 6.88 & -18 & -8 & 0 \\
11 & Right lentiform nucleus & 6.61 & 30 & -2 & 0 \\
12 & Left MFG & 6.43 & -30 & 24 & 30 \\
13 & Right insula & 5.26 & 30 & -28 & 10 \\
14 & Right IPL & 3.78 & 44 & -44 & 40 \\
15 & Left parahippocampal gyrus & 3.50 & -34 & -18 & -20 \\
16 & Right FusG & 3.46 & 36 & -38 & -20 \\
17 & Right lingual gyrus & 4.21 & 8 & -88 & -10 \\
18 & Right STG & 3.40 & 48 & -20 & 0 \\
19 & Left supramarginal gyrus & 3.75 & -46 & -58 & 30 \\
20 & Right supramarginal gyrus & 3.00 & 56 & -60 & 30 \\
21 & Left precuneus & 2.84 & -14 & -64 & 50 \\
\hline
\end{tabular}

FusG, fusiform gyrus; IFG, inferior frontal gyrus; IPL, inferior parietal lobule MeFG, medial frontal gyrus; MEG, magneto-encephalogram; MFG, middle frontal gyrus; STG, superior temporal gyrus; MTG, middle temporal gyrus. left hemisphere included the parahippocampal gyrus and the precuneus. Furthermore, the ASD group showed a bilateral deficit in the supramarginal gyrus. In the $25-60 \mathrm{~Hz}$ band, reduced activity was located to the left inferior frontal gyrus (IFG) and the insula.

Deficits in gamma-band source activity during perceptual organization in participants with ASD

We contrasted gamma-band activity to face versus no-face stimuli between the ASD group versus controls to identify the sources that contributed uniquely to perceptual organization deficits in participants with ASD.

The analysis of the interaction between condition (face vs noface) and group (ASD group vs controls) revealed a complex pattern of hyperactivity and hypoactivity during perceptual organization in the ASD group. In the high gamma band, controls were characterized by enhanced activity relative to the ASD group in a frontoparietal network during perceptual organization, which included the medial and MFG as well as the precuneus (Fig. 7). This hypoactivity in the ASD group was accompanied by increased source activity in posterior regions, including the lingual gyrus, parahippocampal gyrus, and supramarginal gyrus (Fig. 7). See Table 4 for anatomical locations and MNI coordinates.

A reversed pattern of activation differences was found for the low gamma-band range. For the $25-60 \mathrm{~Hz}$ frequency band, the

Table 4. Anatomic locations and MNI coordinates for the sources of high and low gamma-band activity for the interaction between face versus no-face conditions and groups (controls vs participants with ASD)

\begin{tabular}{llrrrr}
\hline & & \multirow{2}{*}{ MEG (MNI) } & \multicolumn{2}{c}{ Coordinates } \\
\cline { 5 - 6 } Cluster & Anatomic location & $t$ value & $x$ & & \multicolumn{1}{c}{$z$} \\
\hline 1 & Right parahippocampal gyrus & -2.41 & 16 & -8 & -20 \\
2 & Right IFG & -2.53 & 40 & 24 & -10 \\
3 & Right MTG & 1.75 & 62 & -32 & 0 \\
4 & Left MeFG & -2.94 & -10 & 38 & 40 \\
5 & Right parahippocampal gyrus & -2.65 & 40 & -34 & -20 \\
6 & Right MeFG & 2.74 & 10 & 38 & -10 \\
7 & Right lingual gyrus & -2.25 & 22 & -72 & -10 \\
8 & Right supramarginal gyrus & -2.35 & 50 & -46 & 30 \\
9 & Right MFG & 2.45 & 30 & 6 & 50 \\
10 & R precuneus & 2.59 & 22 & -52 & 50 \\
\hline
\end{tabular}

IFG, inferior frontal gyrus; MeFG, medial frontal gyrus; MEG, magneto-encephalogram; MFG, middle frontal gyrus; MTG, middle temporal gyrus.

\section{A Controls vs. Participants with ASD, face vs. noface, $25 \mathrm{~Hz}$ to $60 \mathrm{~Hz}$}

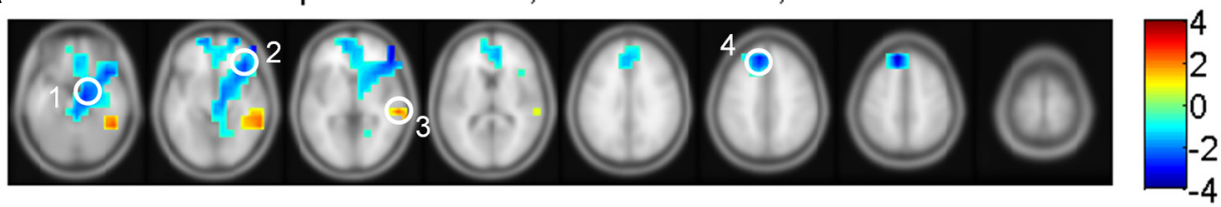

BControls vs. Participants with ASD, face vs. noface, $60 \mathrm{~Hz}$ to $120 \mathrm{~Hz}$

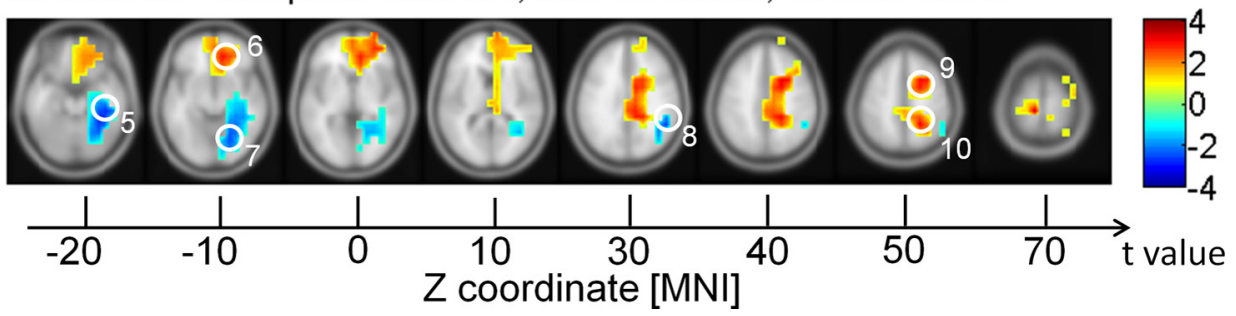

Figure 7. Source power in the high gamma band for the interaction between condition (face vs no-face condition) and group (participants with ASD vs controls). $\boldsymbol{A}, \boldsymbol{B}$, Red clusters represent stronger activation under the two conditions in controls, whereas blue clusters represent stronger activation under the two conditions in participants with ASD. Note that $p$ values are calculated by cluster-based statistical anaylsis. 1, parahippocampal gyrus; 2, IFG; 3, MTG; 4, MeFG; 5, parahippocampal gyrus; 6, MeFG; 7, lingual gyrus; 8, supramarginal gyrus; 9, MFG; 10, precuneus. 
A

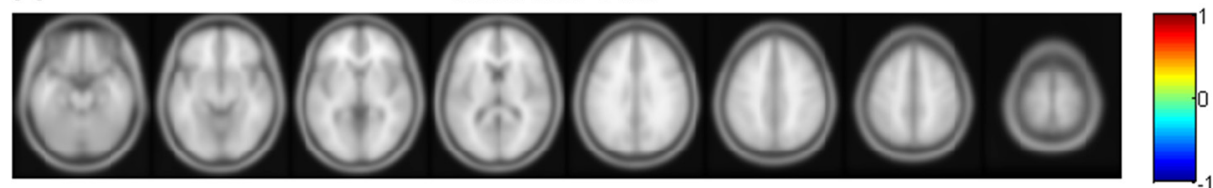

B Controls: Reaction Time

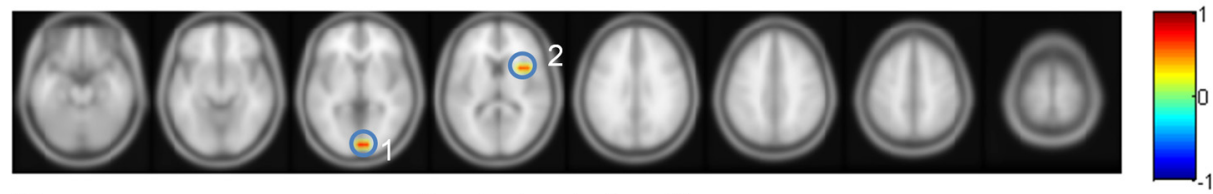

C Controls: Index $A^{\prime}$
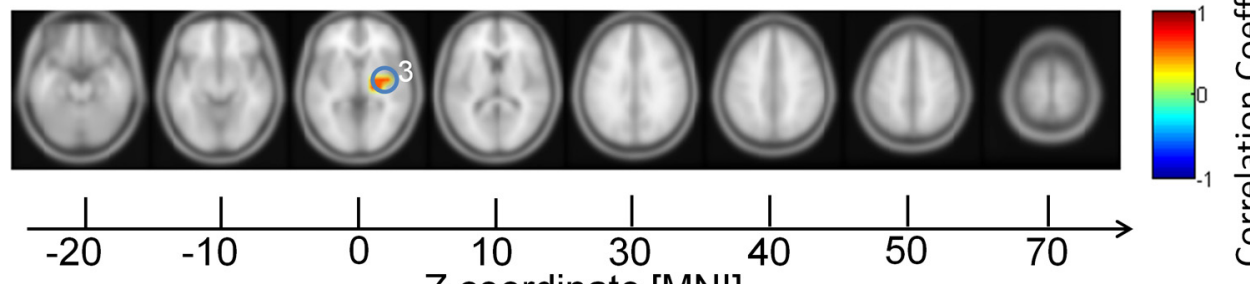

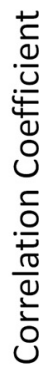

Figure 8. Correlation between behavior data and high gamma power in controls. Correlations with hit rates (A), RTs (B), and discrimination (C). 1, lingual gyrus; 2 and 3, insula.

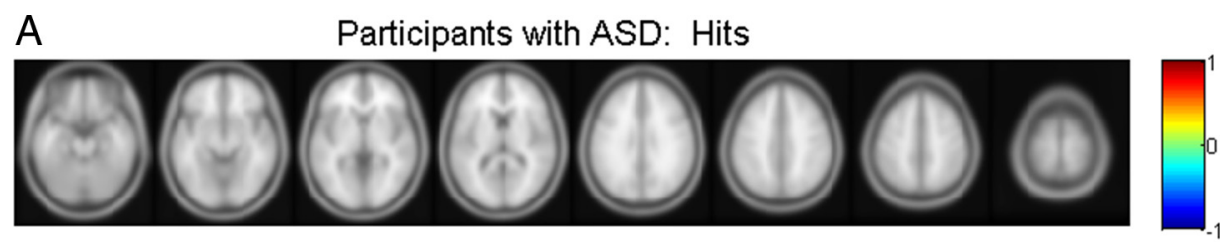

B Participants with ASD: Reaction Time

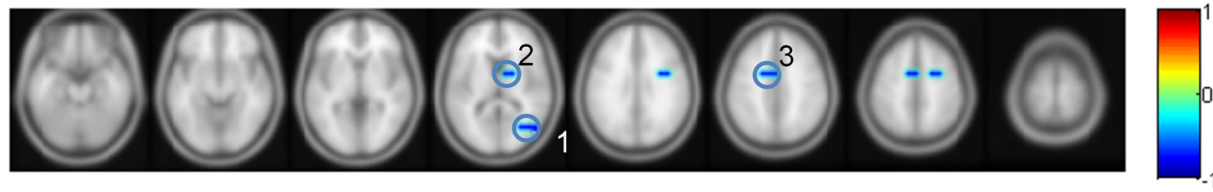

C Participants with ASD: Index $A^{\prime}$

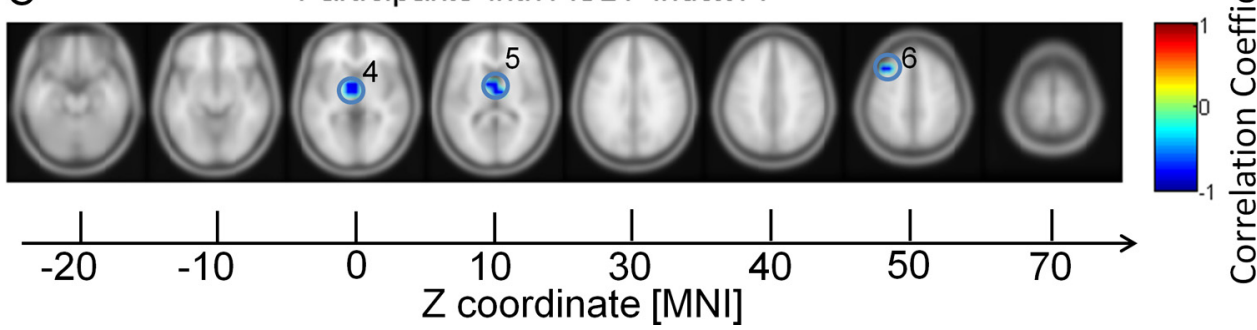

Figure 9. Correlation between behavior data and high gamma power in participants with ASD. Correlations with hit rates $(\boldsymbol{A})$, RTs (B) , and discrimination $(\boldsymbol{C})$. 1, MTG; 2, lentiform nucleus*; 3, cingulate gyrus; 4 and 5, extranuclear*; 6: MFG. *Excluded from analysis.

ASD group was characterized by stronger activity in the inferior and MeFG and in the parahippocampal gyrus during perceptual organization, while controls showed elevated activity in the MTG.

Correlations between behavior and gamma-band source power

Correlations between gamma-band source power and behavioral parameters were computed for controls and the ASD group separately (Fig. 8). For the high gamma band, there was a significant negative correlation between increased gamma-band power in the lingual gyrus and the insula and reduced reaction times in controls. Moreover, there was a significant correlation between increased activity in the insula and the discrimination index $\mathrm{A}^{\prime}$.

Participants with ASD were characterized by a different pattern. Reaction times were negatively correlated with $60-120 \mathrm{~Hz}$ activity in the MTG and cingulate gyrus (Fig. 9). Finally, high gamma-band activity in the MFG correlated negatively with discrimination performance. See Tables 5 and 6 for anatomical locations and MNI coordinates.

\section{Discussion}

We observed that visual processing of Mooney faces in ASD is associated with profound impairments in gamma-band power, 
Table 5. Anatomic locations and MNI coordinates for the correlations between behavioral data and high gamma power in controls

\begin{tabular}{lllllrr}
\hline & & \multirow{2}{*}{ MEG (MNI) } & \multicolumn{2}{c}{ Coordinates } \\
\cline { 5 - 6 } Cluster & Anatomic location & $r$ & $X$ & \multicolumn{1}{l}{$y$} & $Z$ \\
\hline 1 & Right lingual gyrus & 0.63 & 4 & -90 & 0 \\
2 & Right insula & 0.54 & 34 & 10 & 10 \\
3 & Right insula & 0.53 & 42 & -8 & 0 \\
\hline
\end{tabular}

MEG, magneto-encephalogram.

Table 6. Anatomic locations and MNI coordinates for the correlations between behavior data and high gamma power in participants with ASD

\begin{tabular}{llllrr}
\hline & & \multirow{2}{*}{ MEG (MNI) } & \multicolumn{2}{c}{ Coordinates } \\
\cline { 5 - 6 } Cluster & Anatomic location (label) & $r$ & $x$ & \multicolumn{1}{l}{$y$} & $\mathrm{Z}$ \\
\hline 1 & Right MTG & -0.78 & 42 & -70 & 10 \\
2 & Left cingulate gyrus & -0.72 & -16 & 2 & 40 \\
3 & Left MFG & -0.72 & -30 & 20 & 50 \\
\hline
\end{tabular}

MEG, magneto-encephalogram; MFG, middle frontal gyrus; MTG, middle temporal gyrus.

suggesting an impairment in precise timing of neural activity in adult participants with ASD. Moreover, the pattern of dysfunctional gamma-band activity is consistent with previous anatomical and fMRI findings that suggest that frontoposterior networks are differentially affected (Schipul et al., 2011).

Participants with ASD were characterized by impaired behavioral responses to upright Mooney faces, which could reflect dysfunctional configural processing and/or enhanced processing of individual elements (Lahaie et al., 2006). Aberrant detection rates and reaction times to Mooney faces were associated with a reduction of gamma-band power at the sensor level, which started as early as $80 \mathrm{~ms}$ with a decrease in the evoked response over occipitoparietal sensors. Previous studies examining gamma-band power in ASD during complex visual processing in ASD have reported intact evoked responses (Grice et al., 2001; Brown et al., 2005; Milne et al., 2009). The current results suggest that visual processing in ASD is associated with a pronounced deficit in feedforward, transient gamma-band activity, which is supported by event-related potential studies (Milne et al., 2009; Sutherland and Crewther, 2010).

In contrast, induced oscillations are not time locked to the onset of a stimulus but occur with a variable delay between trials, suggesting that these rhythmic fluctuations reflect selfgenerated patterns of neural activity that are important for establishing large-scale synchronization between cortical regions (Varela et al., 2001). Our data suggest that participants with ASDs are characterized by impairments in induced activity in the high gamma-band range as well which was most pronounced over occipitoparietal sensors.

Previous studies examining neural oscillations in ASD had largely focused on oscillatory activity in the 30-60 Hz frequency because oscillations around $\sim 40 \mathrm{~Hz}$ were initially proposed to serve as a mechanism for the binding of spatially distributed responses in vision (Gray et al., 1989). The current data clearly suggest that the main deficit in ASD is in the $60-120 \mathrm{~Hz}$ frequency band. Finally, we found only limited evidence for increased high-frequency activity in the ASD group as previously reported (Brown et al., 2005; Isler et al., 2010; Stroganova et al., 2012) as elevated gamma-band activity was limited to an upregulation of 25-60 Hz activity over frontocentral sensors.

Because only limited conclusions can be drawn from sensor data in regards to the underlying cortical structures that generate neural oscillations, we performed a source localization with a beamforming approach, which allowed the identification of the underlying cortical network. In controls high gamma-band activity in the face condition was found in a distributed network, such as the temporal cortex and IFG, which have been related to face perception and perceptual decision making (Kanwisher et al., 1997; Ploran et al., 2007).

Previous studies have reported conflicting evidence regarding the involvement of the FusG in face-processing deficits in ASD (Pierce et al., 2001; Bird et al., 2006; Dziobek et al., 2010). The current data suggest a pronounced impairment in high gammaband activity in this region, suggesting that $60-120 \mathrm{~Hz}$ power may be a sensitive marker of aberrant processing in networks relevant for face processing. In addition, reduced gamma-band activity in the precuneus suggests that in the ASD group perception of Mooney faces led to a less perceptually integrated object representation as the precuneus has been implicated in the reconstruction of object (or face) representations from fragmentary evidence (Dolan et al., 1997). The reduced "face-like" representation could also explain the decreased activity in the supramarginal gyrus, a brain region that is part of an extended face-processing network, which is particularly involved in the perception of eye-gaze direction (Nummenmaa et al., 2010).

\section{Perceptual organization in ASD involves abnormal modulation of gamma-band activity in cortical networks}

The contrast comparing differences between face and no-face stimuli between the two groups revealed a network in controls, which overlapped with regions identified during the face condition (precuneus, MFG) but included additional activity in the MeFG. Moreover, an additional cluster was detected in the MTG in the $25-60 \mathrm{~Hz}$ band. This frontoposterior network is crucially involved in complex social cognition as suggested by studies that have examined fMRI activity during mentalizing and face perception (Amodio and Frith, 2006).

Perceptual organization of Mooney faces in participants with ASD was characterized by a qualitatively different network because gamma-band sources were focused almost exclusively over posterior regions, notably in the lingual gyrus, parahippocampal gyrus, and supramarginal gyrus. The differential modulation of high gamma-band activity in anterior versus posterior networks is in agreement with both the behavioral phenotype as well as with several anatomical and functional imaging studies that have demonstrated stronger recruitment of sensory regions in ASD (Minshew and Keller, 2010; Schipul et al., 2011) and aberrant connectivity between frontal and posterior regions in EEG data (Coben et al., 2008). In contrast, cortical networks in ASD frequently involve reduced activity in frontal and temporal cortices that are particularly relevant for social cognition, language, and executive functions.

In the current study, elevated high gamma-band activity in visual regions, such as the lingual gyrus, could reflect an emphasis on the "perceptual" qualities of Mooney faces in the ASD group. Moreover, the increased activation in the parahippocampal gyrus suggests that in the ASD group, cortical regions are activated during perceptual organization of face-like stimuli that are not typically recruited during normal brain functioning, possibly indicating a compensatory strategy. This is also supported by the contrast in the low gamma band. In the 25-60 Hz frequency range, participants with ASD showed increased activity relative to controls over frontal regions, which overlapped with reduced gamma-band sources in the $60-120 \mathrm{~Hz}$ frequency, suggesting that cortical networks in ASD generated low gamma-band activity because of dysfunctional circuits in the frontal cortex. 
Finally, we observed a profound alteration in the relationship between gamma-band activity and behavior in the ASD group, which points to decoupling of high-frequency activity from efficient perceptual processing. In controls there was a significant correlation between faster reaction times and increased 60-120 $\mathrm{Hz}$ activation in the lingual gyrus as well as enhanced $60-120 \mathrm{~Hz}$ activity in the insula, which was associated with improved discrimination performance between face and no-face stimuli and faster reaction times. The insula has recently received increased attention as an important brain region during decision making in perceptual paradigms in normal brain functioning (Sterzer and Kleinschmidt, 2010).

The relationship between enhanced high gamma-band activity and behavior was not found in the ASD group. Instead, discrimination performance and reaction times was negatively correlated with $60-120 \mathrm{~Hz}$ activity in several brain regions, including the MTG, cingulate gyrus, and MFG. These data suggest that while high gamma-band activity in frontal and occipital cortices facilitates efficient perceptual processing in controls, this relationship is inversed in ASDs.

\section{Links to the development and neurobiology of ASD}

The pronounced dysfunction in gamma-band activity suggests that changes in the E/I balance may be a pervasive feature of cortical networks in ASD (Rubenstein and Merzenich, 2003; Gogolla et al., 2009), because gamma-band oscillations crucially depend on negative feedback inhibition of pyramidal cells by GABAergic interneurons (Sohal et al., 2009) as well as on glutamate receptor-mediated feedforward excitatory inputs (Traub et al., 2004). Several lines of evidence suggest that this balance is impaired in ASD as indicated by the high incidence of epilepsy (Tuchman and Rapin, 2002) as well as by data showing altered expression of different GABA-receptor subtypes (Fatemi et al., 2002; Oblak et al., 2010) and dysfunctions in glutamatergic neurotransmission (Choudhury et al., 2012).

One hypothesis is that impaired gamma-band activity in ASD is the result of aberrant prenatal and perinatal development that leads to a lasting impairment in neural oscillations. This possibility is supported by the fact that parvalbumin-containing interneurons (PVI) are critical for rhythmic inhibition and, consequently, for the emergence of gamma-band oscillations in cortical networks (Sohal et al., 2009). In addition, PVIs are functionally involved in the induction of critical period plasticity ( $\mathrm{Fa}-$ giolini et al., 2004), suggesting that dysfunctional PVIs in ASD could lead to altered, early brain development and to dysfunctional microcircuit architecture (Gogolla et al., 2009).

\section{Issues for future research}

Future research needs to address to what extent the pattern of behavioral and physiological dysfunctions in a small sample of high-functioning participants with ASD is also associated with ASD during earlier development periods and the result of changes in the $\mathrm{E} / \mathrm{I}$ balance. Moreover, because face-processing deficits in ASD can involve distinct mechanisms and impairments (Lahaie et al., 2006; Webb et al., 2010), intraindividual variability needs to be taken into account through recruiting larger samples, which may allow the detection of subgroups with distinct patterns of behavioral and electrophysiological signatures.

Finally, since the focus in this study was on the investigation of high-frequency activity, relationships of deficits in the high gamma band with low-frequency activity need to be investigated because of the coupling between gamma-band activity and theta- band oscillations during normal brain functioning (Canolty et al., 2006) and to demonstrate the specific contribution of 60-120 $\mathrm{Hz}$ activity toward perceptual impairments in the disorder.

\section{Conclusion}

Our data provide novel evidence for the role of gamma-band activity in visuoperceptual dysfunctions in ASD through demonstrating profound impairments in $60-120 \mathrm{~Hz}$ spectral power in frontoposterior cortical networks in adult participants with ASD. Our data suggest that $60-120 \mathrm{~Hz}$ activity, which underlies perceptual processing in controls, is significantly reduced in ASD and is characterized by a differential relationship with cognition and behavior. While in controls, high gamma-band power has a facilitatory effect, and this relationship is altered in ASD as indicated by the decoupling of $60-120 \mathrm{~Hz}$ activity from behavior and the shift of cortical networks from anterior to posterior brain regions.

\section{References}

Amodio DM, Frith CD (2006) Meeting of minds: the medial frontal cortex and social cognition. Nat Rev Neurosci 7:268-277.

Anderson MJ, ter Braak CJF (2003) Permutation tests for multi-factorial analysis of variance. J Stat Comput Sim 73:85-113.

American Psychiatric Association (2000) DSM-IV-TR. Diagnostic and statistical manual of mental disorders. Washington, DC: American Psychiatric Association.

Baron-Cohen S, Leslie AM, Frith U (1985) Does the autistic child have a "theory of mind"? Cognition 21:37-46.

Bird G, Catmur C, Silani G, Frith C, Frith U (2006) Attention does not modulate neural responses to social stimuli in autism spectrum disorders. Neuroimage 31:1614-1624.

Brown C, Gruber T, Boucher J, Rippon G, Brock J (2005) Gamma abnormalities during perception of illusory figures in autism. Cortex 41:364-376.

Canolty RT, Edwards E, Dalal SS, Soltani M, Nagarajan SS (2006) High gamma power is phase-locked to theta oscillations in human neocortex. Science 313:1626-1628.

Castelli F, Frith C, Happé F, Frith U (2002) Autism, Asperger syndrome and brain mechanisms for the attribution of mental states to animated shapes. Brain 125:1839-1849.

Choudhury PR, Lahiri S, Rajamma U (2012) Glutamate mediated signaling in the pathophysiology of autism spectrum disorders. Pharmacol Biochem Behav 100:841-849.

Coben R, Clarke AR, Hudspeth W, Barry RJ (2008) EEG power and coherence in autistic spectrum disorder. Clin Neurophysiol 119:1002-1009.

Curtin F, Schulz P (1998) Multiple correlations and Bonferroni's correction. Biol Psychiatry 44:775-777.

Delorme A, Makeig S (2004) EEGLAB: an open source toolbox for analysis of single-trial EEG dynamics including independent component analysis. J Neurosci Methods 134:9-21.

Dolan RJ, Fink GR, Rolls E, Booth M, Holmes A (1997) How the brain learns to see objects and faces in an impoverished context. Nature 389:596-599.

Dziobek I, Bahnemann M, Convit A, Heekeren HR (2010) The role of the fusiform-amygdala system in the pathophysiology of autism. Arch Gen Psychiatry 67:397-405.

Fagiolini M, Fritschy JM, Löw K, Möhler H, Rudolph U, Hensch TK (2004) Specific GABAA circuits for visual cortical plasticity. Science 303:1681-1683.

Fatemi SH, Halt AR, Stary JM, Kanodia R, Schulz SC, Realmuto GR (2002) Glutamic acid decarboxylase 65 and $67 \mathrm{kDa}$ proteins are reduced in autistic parietal and cerebellar cortices. Biol Psychiatry 52:805-810.

Freitag CM, Staal W, Klauck SM, Duketis E, Waltes R (2010) Genetics of autistic disorders: review and clinical implications. Eur Child Adolesc Psychiatry 19:169-178.

Fries P (2009) Neuronal gamma-band synchronization as a fundamental process in cortical computation. Annu Rev Neurosci 32:209-224.

Geschwind DH (2011) Genetics of autism spectrum disorders. Trends Cogn Sci 9:409-416.

Gogolla N, Leblanc JJ, Quast KB, Südhof T, Fagiolini M, Hensch TK (2009) 
Common circuit defect of excitatory-inhibitory balance in mouse models of autism. J Neurodev Disord 1:172-181.

Gray CM, König P, Engel AK, Singer W (1989) Oscillatory responses in cat visual cortex exhibit inter-columnar synchronization which reflects global stimulus properties. Nature 338:334-337.

Grice SJ, Spratling MW, Karmiloff-Smith A, Halit H, Csibra G (2001) Disordered visual processing and oscillatory brain activity in autism and Williams syndrome. Neuroreport 12:2697-2700.

Grier JB (1971) Nonparametric indexes for sensitivity and bias: computing formulas. Psychol Bull 75:424-429.

Grützner C, Uhlhaas PJ, Genc E, Kohler A, Singer W, Wibral M (2010) Neuroelectromagnetic correlates of perceptual closure processes. J Neurosci 30:8342-8352.

Happé FG (1996) Studying weak central coherence at low levels: children with autism do not succumb to visual illusions. A research note. J Child Psychol Psychiatry 37:873-877.

Havenith MN, Yu S, Biederlack J, Chen NH, Singer W, Nikolić D (2011) Synchrony makes neurons fire in sequence, and stimulus properties determine who is ahead. J Neurosci 31:8570-8584.

Hill EL (2004) Executive dysfunction in autism. Trends Cogn Sci 8:26-32.

Isler JR, Martien KM, Grieve PG, Stark RI, Herbert MR (2010) Reduced functional connectivity in visual evoked potentials in children with autism spectrum disorder. Clin Neurophysiol 121:2035-2043.

Just MA, Cherkassky VL, Keller TA, Minshew NJ (2004) Cortical activation and synchronization during sentence comprehension in high-functioning autism: evidence of underconnectivity. Brain 127:1811-1821.

Kaiser J, Lutzenberger W (2005) Human gamma-band activity: a window to cognitive processing. Neuroreport 16:207-211.

Kanwisher N, McDermott J, Chun MM (1997) The fusiform face area: a module in human extrastriate cortex specialized for face perception. J Neurosci 17:4302-4311.

Kleinhans NM, Richards T, Sterling L, Stegbauer KC, Mahurin R (2008) Abnormal functional connectivity in autism spectrum disorders during face processing. Brain 131:1000-1012.

Lahaie A, Mottron L, Arguin M, Berthiaume C, Jemel B, Saumier D (2006) Face perception in high-functioning autistic adults: evidence for superior processing of face parts, not for a configural face-processing deficit. Neuropsychology 20:30-41.

Lord C, Rutter M, Le Couteur A (1994) Autism diagnostic interview-revised: a revised version of a diagnostic interview for caregivers of individuals with possible pervasive developmental disorders. J Autism Dev Disord 24:659-685.

Lord C, Risi S, Lambrecht L, Cook EH Jr, Leventhal BL (2000) The autism diagnostic observation schedule-generic: a standard measure of social and communication deficits associated with the spectrum of autism. J Autism Dev Disord 30:205-223.

Maris E, Oostenveld R (2007) Nonparametric statistical testing of EEG- and MEG-data. J Neurosci Methods 164:177-190.

Milne E, Scope A, Pascalis O, Buckley D, Makeig S (2009) Independent component analysis reveals atypical electroencephalographic activity during visual perception in individuals with autism. Biol Psychiatry 65:22-30.

Minshew NJ, Keller TA (2010) The nature of brain dysfunction in autism: functional brain imaging studies. Curr Opin Neurol 23:124-130.

Mooney CM, Ferguson GA (1951) A new closure test. Can J Psychol 5:129-133.

Nieuwenhuis IL, Takashima A, Oostenveld R, Fernández G, Jensen O (2008) Visual areas become less engaged in associative recall following memory stabilization. Neuroimage 40:1319-1327.

Nolte G, Dassios G (2005) Analytic expansion of the EEG lead field for realistic volume conductors. Phys Med Biol 50:3807-3823.

Nummenmaa L, Passamonti L, Rowe J, Engell AD, Calder AJ (2010) Connectivity analysis reveals a cortical network for eye gaze perception. Cereb Cortex 20:1780-1787.

Oblak AL, Gibbs TT, Blatt GJ (2010) Decreased GABA(B) receptors in the cingulate cortex and fusiform gyrus in autism. J Neurochem 114: $1414-1423$.

Papadelis C, Poghosyan V, Fenwick PB, Ioannides AA (2009) MEG's ability to localise accurately weak transient neural sources. Clin Neurophysiol 120:1958-1970.

Pierce K, Müller RA, Ambrose J, Allen G, Courchesne E (2001) Face processing occurs outside the fusiform 'face area' in autism: evidence from functional MRI. Brain 124:2059-2073.

Ploran EJ, Nelson SM, Velanova K, Donaldson DI, Petersen SE, Wheeler ME (2007) Evidence accumulation and the moment of recognition: dissociating perceptual recognition processes using fMRI. J Neurosci 27: 11912-11924.

Raven JC (1948) The comparative assessment of intellectual ability. Br J Psychol 19:137-150.

Rodriguez E, George N, Lachaux JP, Martinerie J, Renault B, Varela FJ (1999) Perception's shadow: long-distance synchronization of human brain activity. Nature 397: 430-433.

Rojas DC, Teale PD, Maharajh K, Kronberg E, Youngpeter K (2011) Transient and steady-state auditory gamma-band responses in first-degree relatives of people with autism spectrum disorder. Mol Autism 2:11.

Rubenstein JL, Merzenich MM (2003) Model of autism: increased ratio of excitation/inhibition in key neural systems. Genes Brain Behav 2:255-267.

Sass1 H, Wittchen HU, Zaudig M, Houben I (2003) Diagnostisches und statistisches Manual psychischer Störungen: Textrevision; DSM-IV-TR (Dt, Bearb.). Göttingen: Hogrefe-Verlag.

Schipul SE, Keller TA, Just MA (2011) Inter-regional brain communication and its disturbance in autism. Front Syst Neurosci 5:10.

Schmötzer G, Rühl D, Thies G, Poustka F (1993) Autismus diagnostisches interview-revision. Frankfurt: J. W. Goethe-University Frankfurt.

Sohal VS, Zhang F, Yizhar O, Deisseroth K (2009) Parvalbumin neurons and gamma rhythms enhance cortical circuit performance. Nature 459:698-702.

Sterzer P, Kleinschmidt A (2010) Anterior insula activations in perceptual paradigms: often observed but barely understood. Brain Struct Funct 214:611-622.

Stroganova TA, Orekhova EV, Prokofyev AO, Tsetlin MM, Gratchev VV (2012) High-frequency oscillatory response to illusory contour in typically developing boys and boys with autism spectrum disorders. Cortex 48:701-717.

Sutherland A, Crewther DP (2010) Magnocellular visual evoked potential delay with high autism spectrum quotient yields a neural mechanism for altered perception. Brain 133:2089-2097.

Traub RD, Bibbig A, LeBeau FE, Buhl EH, Whittington MA (2004) Cellular mechanisms of neuronal population oscillations in the hippocampus in vitro. Annu Rev Neurosci 27:247-278.

Tuchman R, Rapin I (2002) Epilepsy in autism. Lancet Neurol 1:352-358.

Uhlhaas PJ, Singer W (2007) What do disturbances in neural synchrony tell us about autism? Biol Psychiatry 62:190-191.

Uhlhaas PJ, Pipa G, Lima B, Melloni L, Neuenschwander S (2009) Neural synchrony in cortical networks: history, concept and current status. Front Integr Neurosci 3:17.

Van Veen BD, van Drongelen W, Yuchtman M, Suzuki A (1997) Localization of brain electrical activity via linearly constrained minimum variance spatial filtering. IEEE Trans Biomed Eng 44:867-880.

Varela F, Lachaux JP, Rodriguez E, Martinerie J (2001) The brainweb: phase synchronization and large-scale integration. Nat Rev Neurosci 2:229239.

Webb SJ, Jones EJ, Merkle K, Murias M, Greenson J (2010) Response to familiar faces, newly familiar faces, and novel faces as assessed by ERPs is intact in adults with autism spectrum disorders. Int J Psychophysiol 77:106-117.

Wilson TW, Rojas DC, Reite ML, Teale PD, Rogers SJ (2007) Children and adolescents with autism exhibit reduced MEG steady-state gamma responses. Biol Psychiatry 62:192-197. 